\title{
Screening targeted testis-specific genes for molecular assessment of aberrant sperm quality
}

\author{
XUE XIA LIU* ${ }^{*}$ XIAO FANG SHEN* and FU-JUN LIU \\ Central Laboratory, Yantai Yu Huang Ding Hospital, Qingdao University, Yantai, Shandong 264000, P.R. China
}

Received July 4, 2015; Accepted May 9, 2016

DOI: $10.3892 / \mathrm{mmr} .2016 .5434$

\begin{abstract}
Teratospermia is a heterogeneous and complex disorder, which is closely associated with male fertility. Genes and gene products associated with teratospermia may serve as targeted biomarkers that help understand the underlying mechanisms of male infertility; however, systematic information on the subject remains to be elucidated. The present study performed a comparative bioinformatics analysis to identify biomarkers associated with sperm quality, particular focusing on testis-specific biomarkers. A stepwise screening approach identified 1,085 testis/epididymis-specific genes and 3,406 teratospermia-associated genes, resulting in 348 testis-specific genes associated with aberrant sperm quality. These genes were functionally associated with the reproduction process. Gene products corresponding to heat shock protein family A (Hsp70) member 4 like (HSPA4L) and phosphoglycerate kinase 2 were characterized at the cellular level in human testes and ejaculated spermatozoa. HSPA4L expression in sperm was revealed to be associated with sperm quality. The present study provided a novel insight into the understanding of sperm quality, and a potential method for the diagnosis and assessment of sperm quality in the event of male infertility.
\end{abstract}

\section{Introduction}

Increasing environmental pollution and social stressors have led to a decline in male reproductive health, resulting in poor sperm quality and potential male infertility $(1,2)$. Male infertility is a worldwide health concern that affects $>20$ million men. It is a multifactorial disorder, which is triggered by environmental and physiological factors (3). In these cases,

Correspondence to: Dr Fu-Jun Liu, Central Laboratory, Yantai Yu Huang Ding Hospital, Qingdao University, 20 Yudong Road, Yantai, Shandong 264000, P.R. China

E-mail: male_reproduction@163.com

*Contributed equally

Key words: spermatozoa, sperm quality, testis, male infertility, epididymis ejaculated spermatozoa are usually observed to have reduced quantity or poor quality. Diagnosis of male infertility predominantly relies on the traditional evaluation of semen parameters, whereas genetic factors are often overlooked. Previous studies have reported that genetic factors are frequently associated with male fertility, and may provide novel insights into the understanding of male infertility and the assessment of sperm quality (4-6).

Spermatozoa are produced in the testes, and their mRNAs maturate during the spermatogenesis process (7). Sperm mRNAs are believed to be silenced following the release of sperm from the testes; therefore, changes in the expression levels of sperm mRNAs may reflect abnormal spermatogenesis (8). At the protein level, previous studies have compared the proteomic differences between normal and aberrant spermatozoa (9-15). A set of proteins has been identified, which was confirmed to be associated with sperm quality. However, the proteins investigated were limited to the ones in high abundance, due to the technological bias of current proteomics, thus often not fully exploring the underpinning pathways. At the transcriptional level, sperm mRNAs have been used as biomarkers to evaluate sperm quality, and biomarkers associated with abnormal sperm are promising targets for intervention, which may be developed into male contraceptive targets $(16,17)$. In the present study, a systematic comparative analysis was performed in order to screen for testis-specific and teratospermia-associated genes, aiming to identify specific targets associated with sperm quality. The current study provides novel insights into the clinical research of sperm biology and identifies a potential panel of biomarkers for the assessment of sperm quality.

\section{Materials and methods}

Data collection. Gene expression data of normal human tissues (GSE14938) (18) and teratospermia (GSE6967 and GSE6968) (19) were downloaded from the Gene Expression Omnibus (GEO) database (http://www.ncbi.nlm.nih.gov/geo/). The raw data files, which contained the signal intensities and values for every spot on the arrays, were downloaded. The annotation file of the array platform was also downloaded using the GEO query package. Specifically-expressed testis and epididymis genes were identified by statistically comparing the expression of genes from the testis/epididymis with genes from other normal tissues, including bladder, cervix, colon, 
heart, kidney, liver, lung, ovary, prostate, spleen and stomach, using one-tailed t-test with a threshold of $\mathrm{P}<0.001$. Highly expressed genes in the testis and epididymis compared with other tissues, and highly expressed genes in normozoospermia compared with in teratospermia were statistically screened by Student's t-test. $\mathrm{P}<0.01$ was considered to indicate a statistically significant difference. Statistical analysis was performed using SPSS software, version 18.0 (SPSS, Inc., Chicago, IL, USA).

Sample preparation. Testes were used for immunohistochemistry in the present study. Young adult testes were collected from five young fathers (27-33 years old), who had passed away in automobile accidents, had no history of pathology that may affect reproductive functions, and were willing to donate their bodies for medical research while still alive. Donation of organs for medical research was approved by their immediate family members. The elderly samples were collected from five men (78-82 years old) undergoing epididymal excision for prostatic cancer. All procedures were approved by the Ethics Committee of Yu Huang Ding Hospital (Yantai, China). One testis from each donor was processed for protein extraction, whereas the other underwent immunohistochemistry.

In order to perform sperm protein quantification, seminal plasma was collected from two groups consisting of 30 healthy young adults, 30 young men with teratospermia (normal morphology $<4 \%$ ) and 30 young men with asthenozoospermia (progressive motility <32\%) aged between 28 and 32 years. Seminal plasma was collected and processed according to the World Health Organization (WHO) Laboratory Manual for the Examination and Processing of Human Semen (5th edition, 2010). The donations were authorized by the donors with written informed consent according to the regulations and permission of the Ethics Committee of the Yu Huang Ding Hospital (www.who.int/reproductivehealth/publications/ infertility/9789241547789/en/).

Bioinformatics. All proteins were functionally grouped into several categories according to the Gene Ontology Consortium (http://www.geneontology.org/) and literature annotation in PubMed.

Immunohistochemistry. After fixation in Bouin's solution for $12 \mathrm{~h}$, testicular tissue blocks were processed for paraffin embedding by conventional methods and divided into $4 \mu \mathrm{m}$ tissue sections. Antigen retrieval was conducted in a microwave oven for $15 \mathrm{~min}$. Endogenous peroxidases in the tissue sections were inhibited by incubation with $3 \%$ (v/v) $\mathrm{H}_{2} \mathrm{O}_{2}$ for $1 \mathrm{~h}$. Subsequently, 3\% (w/v) bovine serum albumin (BSA; Sigma-Aldrich, St. Louis, MO, USA) in Tris-buffered saline (TBS) was used to block non-specific binding with antibodies at room temperature. Sections were incubated with primary antibodies against HSPA4L (ab81221) and PGK2 (ab186742; Abcam, Cambridge, MA, USA) diluted 1:100 in blocking solution, overnight at $4^{\circ} \mathrm{C}$. The sections were then washed several times with TBS and were incubated with horseradish peroxidase-conjugated anti-rabbit immunoglobulin (Ig)G (ZB-2306; Beijing Zhongshan Golden Bridge Biotechnology Co., Ltd., Beijing, China) at a final dilution of 1:400 for $1 \mathrm{~h}$ at $37^{\circ} \mathrm{C}$. A 3,3'-diaminobenzidine kit (Beijing Zhongshan
Golden Bridge Biotechnology Co., Ltd.) was used to visualize peroxidase activity at the binding sites. Hematoxylin was used to counterstain the sections. Subsequently, the sections were dehydrated and mounted before undergoing bright-field microscopy (DM LB2; Leica Microsystems GmbH, Nussloch, Germany). Pre-immune rabbit $\mathrm{IgG}$ was used as a negative control. Positive immunostaining was used to provide quantitative results. Briefly, images of immunostained sections were analyzed using commercial Image-Pro Plus version 6.0 (Media Cybernetics, Inc., Rockville, MD, USA). The unequal illumination was processed by shading correction, and a reference slide was used to correct the measurement system. A total of 10 fields from each section were scored for each tissue. When the immunostained images were converted to grayscale, a linear combination between the average gray signal intensity and the relative area of positive staining cells was defined as the integrated optical density.

Quantitative assessment of protein expression in spermatozoa. After liquefaction, human ejaculated spermatozoa were washed in phosphate buffered saline (PBS). The sperm pellet was placed on $1 \%(\mathrm{w} / \mathrm{v})$ gelatin-coated slides, air-dried and fixed with ice-cold methanol for $10 \mathrm{~min}$. Slides were blocked for $1 \mathrm{~h}$ at room temperature with $3 \%(\mathrm{w} / \mathrm{v}) \mathrm{BSA}$ in PBS and incubated at $37^{\circ} \mathrm{C}$ for $1 \mathrm{~h}$ with anti-HSPA4L [ab81221; Abcam; diluted 1:50 in PBS containing 3\% (w/v) BSA]. Following three washes with PBS, the corresponding secondary antibody was applied (fluorescein isothiocyanate-labeled goat anti-rabbit IgG; 1:200 in PBS containing 3\% (w/v) BSA; ZF-0311; Beijing Zhingshan Golden Bridge Biotechnology Co., Ltd., Beijing, China). Samples were subsequently washed in PBS and deionized water. Propidium iodide $(0.01 \mathrm{mg} / \mathrm{ml}$; Invitrogen; Thermo Fisher Scientific, Inc.) counterstaining visualized the nuclei. Quantitative assessment of protein expression in spermatozoa was achieved by scanning confocal microscopy. Slides were systematically examined at a magnification of $\mathrm{x} 400$ according to the WHO manual 2010. Inspection was performed in sequence until a total of 200 spermatozoa had been assessed. Using LSM 510 META software (LSM 5 version 3.2; Carl Zeiss AG, Oberchoken, Germany) the fluorescence intensity value per stained cell was calculated automatically by subtracting the background fluorescence intensity, which was determined by scanning a sperm-free area. SPSS software, version 18.0 (SPSS, Inc.) was used to perform Pearson's correlation coefficient analysis. $\mathrm{P}<0.05$ was considered to indicate a statistically significant difference.

\section{Results}

Identification of significantly expressed genes in the testis and epididymis. By comparing gene expression data sets from 11 normal tissues with that of the testis and epididymis, a total of 1,085 significantly expressed genes were identified in the testis and epididymis. From this total, 770 genes exhibited greater expression in the testis, and 97 genes in the epididymis. These 867 genes were used as background data for subsequent screening (Fig. 1).

Identification of poorly expressed genes in teratospermia. The reduced expression of certain genes in teratospermia 


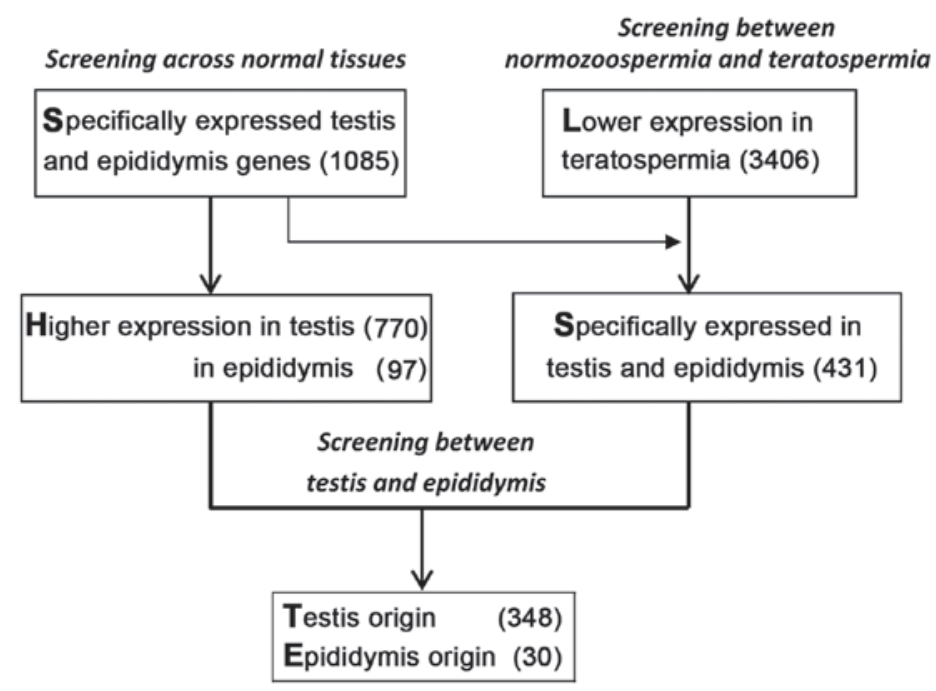

Figure 1. Flowchart of identification of key teratospermia-associated genes.

Table I. Chromosomal distribution of human teratospermia-associated genes and testis/epididymis specific genes.

\begin{tabular}{|c|c|c|c|c|c|c|c|}
\hline \multirow[b]{2}{*}{ Chromosome } & \multirow{2}{*}{$\begin{array}{c}\text { Chromosomal } \\
\text { size (Mbp) }\end{array}$} & \multicolumn{2}{|c|}{ Observed } & \multicolumn{2}{|c|}{ Expected } & \multicolumn{2}{|c|}{ Ratio } \\
\hline & & $\mathrm{Sp}$ & $\mathrm{TE}$ & $\mathrm{Sp}$ & $\mathrm{TE}$ & $\mathrm{Sp}$ & TE \\
\hline 1 & 251 & 367 & 100 & 276 & 88 & 1.33 & 1.14 \\
\hline 2 & 243 & 262 & 71 & 267 & 85 & 0.98 & 0.84 \\
\hline 3 & 198 & 229 & 62 & 218 & 69 & 1.05 & 0.90 \\
\hline 4 & 191 & 140 & 38 & 210 & 67 & 0.67 & 0.57 \\
\hline 5 & 180 & 180 & 39 & 198 & 63 & 0.91 & 0.62 \\
\hline 6 & 171 & 187 & 58 & 188 & 60 & 0.99 & 0.97 \\
\hline 7 & 159 & 146 & 45 & 175 & 56 & 0.83 & 0.81 \\
\hline 8 & 146 & 139 & 32 & 161 & 51 & 0.87 & 0.63 \\
\hline 9 & 141 & 128 & 43 & 155 & 49 & 0.82 & 0.87 \\
\hline 10 & 136 & 152 & 41 & 150 & 48 & 1.02 & 0.86 \\
\hline 11 & 135 & 183 & 59 & 149 & 47 & 1.23 & 1.25 \\
\hline 12 & 134 & 197 & 45 & 147 & 47 & 1.34 & 0.96 \\
\hline 13 & 115 & 81 & 22 & 127 & 40 & 0.64 & 0.55 \\
\hline 14 & 107 & 117 & 37 & 118 & 37 & 0.99 & 0.99 \\
\hline 15 & 102 & 124 & 34 & 112 & 36 & 1.10 & 0.95 \\
\hline 16 & 92 & 133 & 55 & 101 & 32 & 1.31 & 1.71 \\
\hline 17 & 81 & 161 & 62 & 89 & 28 & 1.81 & 2.19 \\
\hline 18 & 78 & 60 & 26 & 86 & 27 & 0.70 & 0.95 \\
\hline 19 & 59 & 110 & 55 & 65 & 21 & 1.69 & 2.67 \\
\hline 20 & 63 & 94 & 36 & 69 & 22 & 1.36 & 1.63 \\
\hline 21 & 48 & 39 & 10 & 53 & 17 & 0.74 & 0.60 \\
\hline 22 & 51 & 54 & 29 & 56 & 18 & 0.96 & 1.63 \\
\hline$X$ & 155 & 117 & 74 & 171 & 54 & 0.69 & 1.37 \\
\hline $\mathrm{Y}$ & 59 & 6 & 9 & 65 & 21 & 0.09 & 0.44 \\
\hline
\end{tabular}

Ratio, the ratio of the number of observed testis-specific genes to the expected number. The number of expected genes was calculated according to chromosome size, on the assumption that testicular genes are uniformly distributed throughout the genome; chromosome size is obtained from the human genome. Sp, teratospermia-associated genes; TE, testis/epididymis specific genes.

may be a potential biomarker for evaluating sperm quality. By comparing the differential gene expression in normal spermatozoa and teratospermia, 3,406 genes exhibited significantly lower expression in teratospermia. From this 
total 1,087 and 750 genes had higher expression levels in the testis and epididymis, respectively. When specific testis and epididymis genes were compared, it was determined that 431 testis and epididymis specific genes were poorly expressed in teratospermia. Of these genes, highly expressed genes in the testis were termed to be of testis origin, and highly expressed ones in the epididymis were termed to be of epididymis origin. Finally, 348 testis origin genes and 30 epididymis origin genes were obtained, respectively (Fig. 1).

Specific genes in the testis and epididymis are target genes that are significantly associated with spermatogenesis and sperm maturation. Chromosomal distribution analysis of the 1,085 identified genes highlighted that the genes were mainly expressed on chromosomes $17($ ratio=2.19) and $19($ ratio=2.67), whereas less were expressed on chromosomes Y (ratio=0.44) and 13 (ratio=0.55) $($ Table I). The gene ontology analysis demonstrated that proteins corresponding to these genes were primarily involved in functions of metabolism (22\%), structure (16\%), protease/protease inhibitor (16\%), as well as the major functions of transcription (11\%) and signal transduction (10\%) (Fig. 2A). Over-representative analysis indicated that these proteins were significantly involved in the biological processes of cell cycle, mitosis and spermatogenesis (Table II).

Poorly expressed genes in teratospermia may be associated with abnormal spermatogenesis, which in turn may be involved in poor sperm quality in vitro. Poorly expressed genes in teratospermia were mainly distributed at chromosomes 17 $($ ratio $=1.81)$ and 19 (ratio=1.69), and less so at chromosomes $\mathrm{Y}$ $($ ratio $=0.09)$ and $13($ ratio $=0.64)($ Table I). All of the genes were broadly categorized into 13 functional clusters, as presented in Fig. 2B. The proteins corresponding to these genes were primarily involved in metabolism $(26 \%)$, whereas signal transduction $(12 \%)$ and structure $(10 \%)$ were also main functions. In addition, these proteins were significantly involved in biological processes of cell cycle, mRNA processing, protein metabolic process and translation (Table II).

The final 336 key proteins contributed to metabolism (19\%), reproduction (16\%) and protease/protease inhibitor (13\%) (Fig. 2C). These genes were mainly involved in cell cycle, meiosis and mitosis (Table II).

Immunohistochemical localization. From the 348 key proteins, the chaperone protein, heat shock protein family A (Hsp70) member 4 like (HSP4AL) and metabolism protein, phosphoglycerate kinase 2 (PGK2), were selected for cellular localization investigation in the testis and epididymis tissue samples obtained from young and aged men. As presented in Fig. 3, HSPA4L was primarily expressed in round spermatids, whereas PGK2 was primarily expressed in spermatocytes and round spermatids. The proteins exhibited higher expression in the testis compared with the epididymis. In addition, the two proteins had lower expression levels in the testis samples from the aged men, compared with the younger men, thus suggesting there may be an age-related pattern of expression.

Association of HSPA4L expression with sperm functions. Immunofluorescent analysis indicated that HSPA4L was

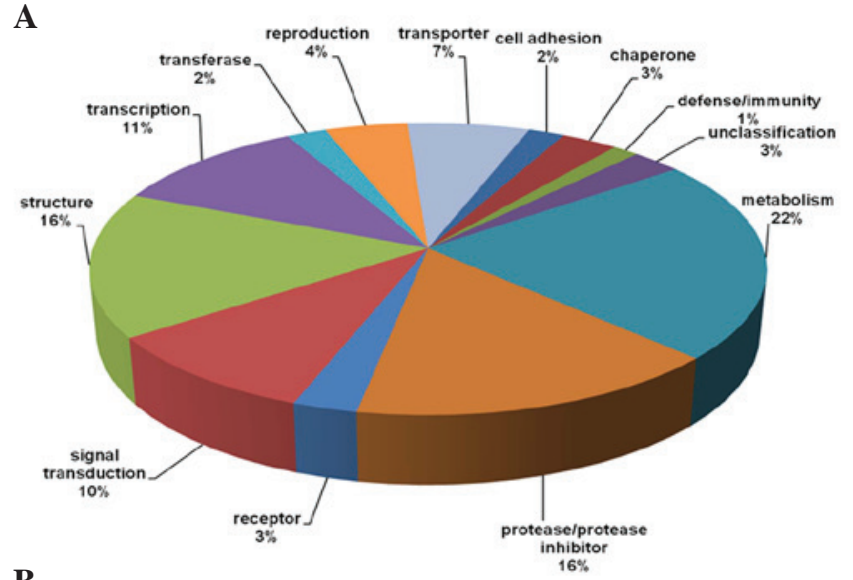

B
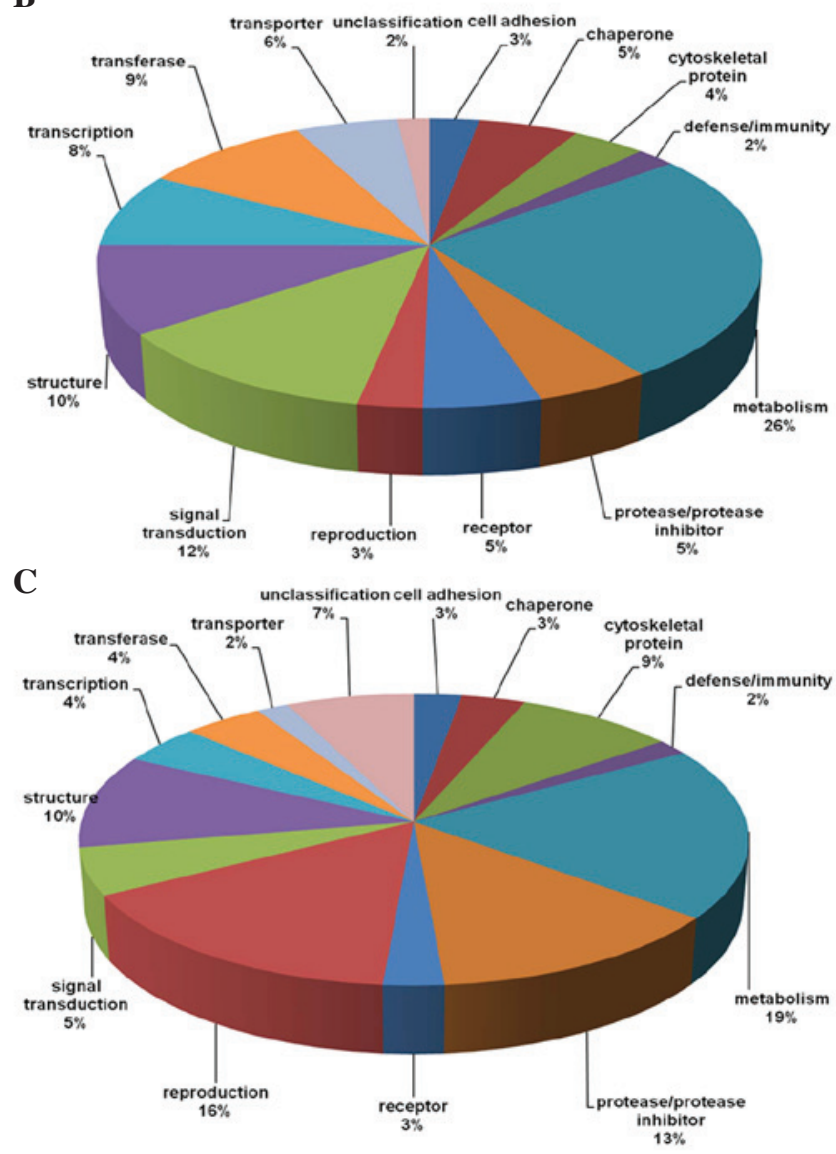

Figure 2. Broad functional analysis of proteins corresponding to (A) testis/epididymis-specific genes, (B) teratospermia-associated genes and (C) key targeted testis-specific teratospermia-associated genes.

located on the sperm acrosome. Quantification of protein localization was performed using confocal scanning microscopy, which indicated that the percentage of sperm with positive staining for HSPA4L and fluorescence intensity was lower in patients with asthenozoospermia compared with normal young adults (Fig. 4).

There was a significant correlation between quantification of HSPA4L in ejaculated spermatozoa and progressive sperm motility. Higher correlation coefficients were observed between staining percentage and intensity and progressive sperm motility and sperm number in the sperm samples from patients with asthenozoospermia (Table III). 
Table II. Over-representative functional analysis of testis/epididymis specific genes and key teratospermia genes.

\begin{tabular}{|c|c|c|}
\hline Biological process & Number & P-value \\
\hline \multicolumn{3}{|l|}{ Sperm proteins } \\
\hline Cell cycle & 454 & $8.04 \times 10^{-12}$ \\
\hline Cellular component biogenesis & 46 & $4.38 \times 10^{-2}$ \\
\hline Chromosome segregation & 66 & $3.98 \times 10^{-2}$ \\
\hline DNA metabolic process & 145 & $1.08 \times 10^{-3}$ \\
\hline Generation of metabolites and energy & 96 & $1.61 \times 10^{-2}$ \\
\hline Intracellular protein transport & 392 & $1.59 \times 10^{-5}$ \\
\hline Mitosis & 184 & $4.06 \times 10^{-6}$ \\
\hline mRNA processing & 191 & $1.87 \times 10^{-14}$ \\
\hline Oxidative phosphorylation & 29 & $1.65 \times 10^{-2}$ \\
\hline Protein metabolic process & 934 & $8.49 \times 10^{-31}$ \\
\hline Protein transport & 396 & $1.77 \times 10^{-5}$ \\
\hline RNA localization & 43 & $1.79 \times 10^{-4}$ \\
\hline Translation & 230 & $1.33 \times 10^{-31}$ \\
\hline \multicolumn{3}{|l|}{ Testis and epididymis proteins } \\
\hline Cell cycle & 131 & $2.32 \times 10^{-11}$ \\
\hline Chromosome segregation & 23 & $5.70 \times 10^{-3}$ \\
\hline Fertilization & 13 & $3.98 \times 10^{-2}$ \\
\hline Gamete generation & 49 & $2.60 \times 10^{-3}$ \\
\hline Mitosis & 61 & $7.73 \times 10^{-8}$ \\
\hline Regulation of catalytic activity & 81 & $2.45 \times 10^{-2}$ \\
\hline Reproduction & 55 & $1.67 \times 10^{-3}$ \\
\hline RNA localization & 14 & $1.06 \times 10^{-2}$ \\
\hline Spermatogenesis & 30 & $1.19 \times 10^{-4}$ \\
\hline \multicolumn{3}{|l|}{ Final target proteins } \\
\hline Cell cycle & 59 & $3.01 \times 10^{-6}$ \\
\hline Meiosis & 9 & $3.47 \times 10^{-2}$ \\
\hline Mitosis & 31 & $1.28 \times 10^{-5}$ \\
\hline
\end{tabular}

\section{Discussion}

Spermatozoa are produced in the testes and mature in the epididymis; therefore, the testis and epididymis are the two critical organs responsible for the formation of functional ejaculated sperm (20). This process includes two major biological processes: Spermatogenesis and sperm maturation. Complex internal pathways are involved in these processes via the interactions of various functional proteins. Notably, various testis or epididymis-specific proteins are important for the maintenance or modification of sperm functions $(21,22)$. Suboptimal expression levels of these proteins may alter sperm functions; therefore, these proteins may serve as potential biomarkers to assess sperm quality.

The present study used a novel comparative bioinformatics analysis to identify tissue-specific genes and genes associated with abnormal sperm. It was hypothesized that genes with lower expression levels, particularly testis/epididymis-specific genes in teratospermia may be considered promising biomarkers for the assessment of sperm quality, which may provide novel insights for male infertility research. When

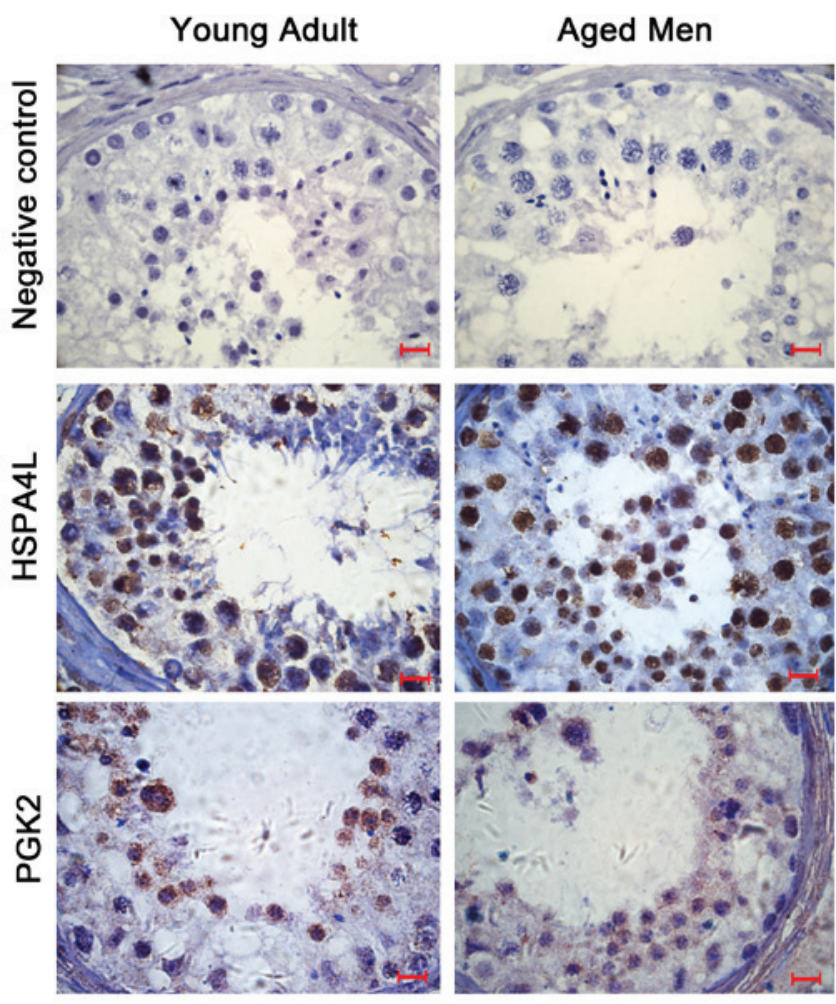

Figure 3. Cellular localization of heat shock protein family A (Hsp70) member 4 like (HSPA4L) and phosphoglycerate kinase 2 (PGK2) in human testis by immunohistochemistry. Scale bar, $20 \mu \mathrm{m}$.

normal tissues were compared with testis/epididymis tissues, 1,085 genes were determined to be specifically expressed in the testis and epididymis. These genes were used as background data to screen for sperm quality-associated genes that were associated with spermatogenesis or sperm maturation. These identified genes were mainly sourced from chromosomes 17 and 19. Ontological analysis indicated that gene products corresponding to these genes functioned predominantly in metabolism, structure and protease/protease inhibition. Over-representative analysis determined the significant biological processes. It was demonstrated that chromosome segregation, fertilization, gamete generation, mitosis and spermatogenesis were the established spermatogenesis-associated functions. Proteins involved in chromosome segregation may be responsible for human infertility (23). Proteins involved in functions of mitosis may be important for the proliferation of germ cells and the differentiation of haploid spermatids (24).

A large number of teratospermia-associated genes were identified in the present study, which primarily contributed to the regulation of metabolism, signal transduction and structure. Corresponding to functions of testis/epididymis-specific genes, a greater proportion of the genes were involved in the functions of transferase, transporter, cell adhesion, chaperone, defense/immunity and receptors. These genes were mainly involved in intercellular biological processes and were ascribed to testicular gene expression. Further screening detected the genes that overlapped with testis/epididymis-specific genes, and 348 testis-specific genes were identified, which were considered promising targets for future clinical research. Notably, the majority of these genes were associated with 


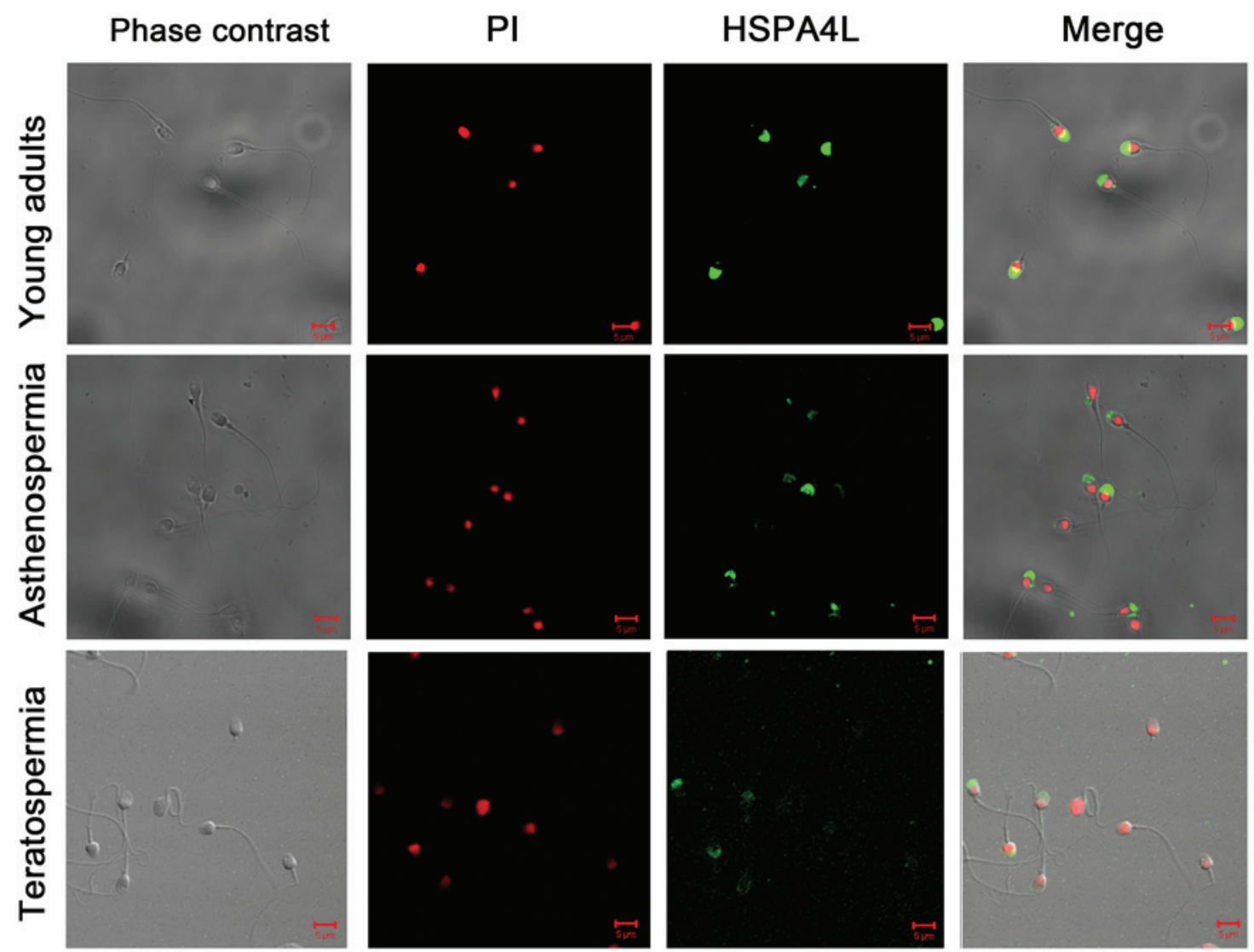

Figure 4. Immunofluorescent quantitative localization of heat shock protein family A (Hsp70) member 4 like (HSPA4L) in ejaculated spermatozoa from young adult men, and patients with asthenozoospermia and teratospermia. PI, propidium iodide.

reproductive activities, and were involved in the biological processes of cell cycle, meiosis and mitosis.

A recent study reported that various key genes are responsible for teratospermia (4). These genes were included in the dataset of the present study. Due to abnormal meiotic segregation, sperm heads have aneuploid composition leading to macrozoospermia. Aurora kinase $\mathrm{C}$ is involved in meiosis and spermatogenesis, and mutation of this gene is responsible for macrozoospermia (25). In addition, genetic mutations in spermatogenesis associated 16 , protein interacting with $C$ kinase 1 and dpy-19 like 2 are associated with globozoospermia (26-28). Other genes were initially reported to be associated with the formation of teratospermia, which require further investigation. Specifically, testis-specific genes may be considered potential biomarkers for the assessment of ejaculated sperm quality. In the present study, the gene products of HSPA4L and PGK2 were characterized in human testes from elderly men, which may reflect age or androgen-associated alterations in their expression. Further quantification of HSPA4L in normal and poor quality sperm was performed by immunofluorescence, and the results indicated that the expression of HSPA4L in the sperm may be affected by testicular alteration and was closely associated with sperm quality. It is possible that these genes and their products may be potential targets for the assessment of sperm quality, and warrant further investigation.

In conclusion, 336 testis-specific genes were determined to be associated with teratospermia, which were also
Table III. Relationship between heat shock protein family A (Hsp70) member 4 like quantification in ejaculated sperm and progressive sperm motility and total sperm counts in semen samples from young adults and patients with asthenozoospermia.

\begin{tabular}{lcccccc}
\hline & \multicolumn{2}{c}{ Stained (\%) } & & \multicolumn{2}{c}{ Intensity } \\
\cline { 2 - 3 } Parameter & $\mathrm{r}$ & $\mathrm{P}$ & & $\mathrm{r}$ & $\mathrm{P}$ \\
\hline Progressive sperm motility & & & & & \\
$\quad \begin{array}{l}\text { Young adults } \\
\text { Asthenozoospermia }\end{array}$ & 0.522 & $\mathrm{P}<0.05$ & & 0.397 & $\mathrm{NS}$ \\
Total sperm number & & & & & & \\
$\quad$ Young adults & 0.431 & $\mathrm{NS}$ & & 0.501 & $\mathrm{P}<0.05$ \\
Asthenozoospermia & 0.524 & $\mathrm{P}<0.05$ & & 0.638 & $\mathrm{P}<0.05$
\end{tabular}

Linear regression correlation coefficients from confocal scanning microscopic evaluation, and statistical significance of coefficients. NS, not significant.

potentially associated with sperm quality and may be used as promising biomarkers for male infertility research. HSPA4L was determined to be associated with aberrant sperm quality. The biomarkers identified in the present study warrant further investigation and may be used as a panel of potential biomarkers for the assessment of sperm quality. 


\section{Acknowledgements}

The present study was supported by grants from the National Natural Science Foundation of China (grant no. 81300533), the Shandong Provincial Natural Science Foundation, China (grant no. ZR2013HQ002), Yantai Science and Technology Program (grant no. 2015WS002) and Youth Science Foundation of Yantai Yu Huang Ding Hospital, China (grant no. 201401).

\section{References}

1. Olea N and Fernandez MF: Chemicals in the environment and human male fertility. Occup Environ Med 64: 430-431, 2007.

2. Rockliff HE, Lightman SL, Rhidian E, Buchanan H, Gordon U and Vedhara $\mathrm{K}$ : A systematic review of psychosocial factors associated with emotional adjustment in in vitro fertilization patients. Hum Reprod Update 20: 594-613, 2014.

3. Manfo FP, Nantia EA and Mathur PP: Effect of environmental contaminants on mammalian testis. Curr Mol Pharmacol 7: $119-135,2014$

4. Coutton C, Escoffier J, Martinez G, Arnoult C and Ray PF: Teratozoospermia: Spotlight on the main genetic actors in the human. Hum Reprod Update 21: 455-485, 2015.

5. De Braekeleer M, Nguyen MH, Morel F and Perrin A: Genetic aspects of monomorphic teratozoospermia: A review. J Assist Reprod Genet 32: 615-623, 2015.

6. Brahem S, Elghezal H, Ghédir H, Landolsi H, Amara A, Ibala S, Gribaa M, Saad A and Mehdi M: Cytogenetic and molecular aspects of absolute teratozoospermia: Comparison between polymorphic and monomorphic forms. Urology 78: 1313-1319, 2011.

7. Hamatani T: Human spermatozoal RNAs. Fertil Steril 97: 275-281, 2012.

8. Dadoune JP: Spermatozoal RNAs: What about their functions? Microsc Res Tech 72: 536-551, 2009.

9. Zhao C, Huo R, Wang FQ, Lin M, Zhou ZM and Sha JH: Identification of several proteins involved in regulation of sperm motility by proteomic analysis. Fertil Steril 87: 436-438, 2007.

10. Martinez-Heredia J, de Mateo S, Vidal-Taboada JM, Ballescà JL and Oliva R: Identification of proteomic differences in asthenozoospermic sperm samples. Hum Reprod 23: 783-791, 2008.

11. Chan CC, Shui HA, Wu CH, Wang CY, Sun GH, Chen HM and Wu GJ: Motility and protein phosphorylation in healthy and asthenozoospermic sperm. J Proteome Res 8: 5382-5386, 2009.

12. Siva AB, Kameshwari DB, Singh V, Pavani K, Sundaram CS, Rangaraj N, Deenadayal M and Shivaji S: Proteomics-based study on asthenozoospermia: Differential expression of proteasome alpha complex. Mol Hum Reprod 16: 452-462, 2010.

13. Shen S, Wang J, Liang J and He D: Comparative proteomic study between human normal motility sperm and idiopathic asthenozoospermia. World J Urol 31: 1395-1401, 2013.
14. Parte PP, Rao P, Redij S, Lobo V, D'Souza SJ, Gajbhiye R and Kulkarni V: Sperm phosphoproteome profiling by ultra performance liquid chromatography followed by data independent analysis (LC-MS (E)) reveals altered proteomic signatures in asthenozoospermia. J Proteomics 75: 5861-5871, 2012.

15. Amaral A, Paiva C, Attardo Parrinello C, Estanyol JM, Ballescà JL, Ramalho-Santos $\mathrm{J}$ and Oliva R: Identification of proteins involved in human sperm motility using high-throughput differential proteomics. J Proteome Res 13: 5670-5684, 2014.

16. Garrido N, García-Herrero S and Meseguer M: Assessment of sperm using mRNA microarray technology. Fertil Steril 99: 1008-1022, 2013.

17. Feugang JM, Rodriguez-Osorio N, Kaya A, Wang H, Page G, Ostermeier GC, Topper EK and Memili E: Transcriptome analysis of bull spermatozoa: Implications for male fertility. Reprod Biomed Online 21: 312-324, 2010.

18. She X, Rohl CA, Castle JC, Kulkarni AV, Johnson JM and Chen R: Definition, conservation and epigenetics of housekeeping and tissue-enriched genes. BMC Genomics 10: 269, 2009.

19. Platts AE, Dix DJ, Chemes HE, Thompson KE, Goodrich R, Rockett JC, Rawe VY, Quintana S, Diamond MP, Strader LF and Krawetz SA: Success and failure in human spermatogenesis as revealed by teratozoospermic RNAs. Hum Mol Genet 16: 763-773, 2007.

20. Hinton BT and Cooper TG: The epididymis as a target for male contraceptive development. Handb Exp Pharmacol 198: 117-137, 2010.

21. Gupta GS: LDH-C4: A target with therapeutic potential for cancer and contraception. Mol Cell Biochem 371: 115-127, 2012.

22. Dacheux JL and Dacheux F: New insights into epididymal function in relation to sperm maturation. Reproduction 147: R27-R42, 2013.

23. Kogo H, Kowa-Sugiyama H, Yamada K, Bolor H, Tsutsumi M, Ohye T, Inagaki $\mathrm{H}$, Taniguchi M, Toda $\mathrm{T}$ and Kurahashi $\mathrm{H}$ : Screening of genes involved in chromosome segregation during meiosis I: Toward the identification of genes responsible for infertility in humans. J Hum Genet 55: 293-299, 2010.

24. Grimes SR: Testis-specific transcriptional control. Gene 343 $11-22,2004$.

25. Ounis L, Zoghmar A, Coutton C, Rouabah L, Hachemi M, Martinez D, Martinez G, Bellil I, Khelifi D, Arnoult C, et al: Mutations of the aurora kinase $\mathrm{C}$ gene causing macrozoospermia are the most frequent genetic cause of male infertility in Algerian men. Asian J Androl 17: 68-73, 2015.

26. Karaca N, Yilmaz R, Kanten GE, Kervancioglu E, Solakoglu S and Kervancioglu ME: First successful pregnancy in a globozoospermic patient having homozygous mutation in SPATA16. Fertil Steril 102: 103-107, 2014

27. Koscinski I, Elinati E, Fossard C, Redin C, Muller J, Velez de la Calle J, Schmitt F, Ben Khelifa M, Ray PF, Kilani Z, et al: DPY19L2 deletion as a major cause of globozoospermia. Am J Hum Genet 88: 344-350, 2011.

28. Li X, Mao Z, Wu M and Xia J: Rescuing infertility of Pick1 knockout mice by generating testis-specific transgenic mice via testicular infection. Sci Rep 3: 2842, 2013. 